Pacific

Journal of

Mathematics

ON THE BEHAVIOUR OF $\infty$-HARMONIC FUNCTIONS ON SOME SPECIAL UNBOUNDED DOMAINS

TILAK BHATTACHARYA

Volume $219 \quad$ No. 2

April 2005 


\title{
ON THE BEHAVIOUR OF $\infty$-HARMONIC FUNCTIONS ON SOME SPECIAL UNBOUNDED DOMAINS
}

\author{
TILAK BHATTACHARYA
}

\begin{abstract}
We study nonnegative $\infty$-harmonic functions defined on unbounded domains, in particular the half-space and the exterior of the unit closed ball. We prove that if such a function $u$ vanishes continuously on the boundary then in the first case $u$ is affine, and in the second case $u$ is radial and linear. We also discuss growth rates in an infinite strip.
\end{abstract}

\section{Introduction and statements of results}

We study nonnegative $\infty$-harmonic functions on unbounded domains with special geometry, in particular the half-space and the exterior of the unit closed ball. We consider functions that vanish on the boundaries while their behaviour at infinity is left unspecified. One may view this work as a step towards understanding the kind of growth rates possible for infinity-harmonic functions on unbounded domains. An analogous result appears in [Crandall et al. 2001], where it is shown that an $\infty$-harmonic function bounded below by a plane is affine. This is related to the conjecture that globally Lipschitz $\infty$-harmonic functions on $\mathbb{R}^{n}$ are affine; however we do not attempt to prove this. The restriction on the sign plays a strong role in this work and has been critical in obtaining estimates for growth rates. It is unclear what happens if this restriction is removed.

Let $u=u(x)$ be an $\infty$-harmonic function defined on a (possibly unbounded) domain $\Omega \subset \mathbb{R}^{n}$, for $n \geq 2$. That is, $u$ solves

$$
\Delta_{\infty} u(x)=\sum_{i, j=1}^{n} \frac{\partial u}{\partial x_{i}} \frac{\partial u}{\partial x_{j}} \frac{\partial^{2} u}{\partial x_{i} \partial x_{j}}=0 \quad \text { for } x \in \Omega
$$

in the viscosity sense. We refer to [Bhattacharya 2002; 2004, Crandall and Evans 2001; Crandall et al. 1992; 2001] for definitions. For the most part we assume that

MSC2000: 35J60, 35J70.

Keywords: $\infty$-harmonic functions, viscosity solutions, growth rates, unbounded domains. This research is partially supported by a fund from the NSERC. 
$u(x) \geq 0$ for $x \in \Omega$, that the boundary $\partial \Omega$ is smooth, and that $u$ is continuous up to $\partial \Omega$. Let $O$ denote the origin in $\mathbb{R}^{n}$, and for $x=\left(x_{1}, x_{2}, \ldots, x_{n}\right) \in \mathbb{R}^{n}$, set

$$
|x|=\sqrt{\sum_{i=1}^{n} x_{i}^{2}}
$$

Theorem 1.1 (The infinite half-space). Let $\Omega=\left\{x \in \mathbb{R}^{n}: x_{n}>0\right\}$ be the infinite half-space. Suppose $u(x) \geq 0$ is $\infty$-harmonic in $\Omega$ and vanishes continuously on the hyperplane $\left\{x_{n}=0\right\}$. Then either $u(x)=0$ for all $x \in \Omega$, or there exists a positive constant $K>0$ such that $u(x)=K x_{n}$ for all $x \in \Omega$.

In this case, the sign restriction leads to linear growth rate in $\Omega$. This also holds when $\Omega$ is the exterior of a ball. In both cases, linear growth rate implies global Lipschitz continuity. The truth of the conjecture mentioned earlier would then imply Theorem 1.1. Solutions with unrestricted sign may have faster growth rates as demonstrated by the well known example

$$
u(x, y)=|x|^{4 / 3}-|y|^{4 / 3}
$$

on $\mathbb{R}^{2}$, in the half planes bounded by $|x|=|y|$. It is not clear whether a growth rate faster than $\frac{4}{3}$ is possible in general, or whether the imposition of a growth rate of $\frac{4}{3}$ would imply that $u$ is of this type.

Let $B(1, O)$ be the unit open ball in $\mathbb{R}^{n}$, centered at $O$, and let $\Omega=\mathbb{R}^{n} \backslash \bar{B}(1, O)$, where $\bar{B}(1, O)$ denotes the closure of $B(1, O)$.

Theorem 1.2 (The exterior of a ball). Let $u \geq 0$ be $\infty$-harmonic in $\Omega$. Suppose that $u$ vanishes continuously on $\partial B(1, O)$. Then either $u(x)=0$ for all $x \in \Omega$, or there exists a positive constant $K$ such that $u(x)=K(|x|-1)$ for all $x \in \Omega$.

While solutions are globally Lipschitz continuous, Theorem 1.2 would not follow from the conjecture mentioned earlier. It is unclear if a faster growth rate is possible when the sign restriction is removed. It would also be interesting to know if Theorems 1.1 and 1.2 would follow for solutions with unrestricted sign but with linear growth rate.

We also discuss the case of the infinite strip $\left\{x: 0<x_{n}<1\right\}$ and show that any nontrivial solution $u(r)$ grows faster than any integral power of $r$, where $r$ is the distance from the $x_{n}$-axis. However, it is not clear if nontrivial solutions exist (see Section 5). In this work, we make considerable use of the properties proven in [Bhattacharya 2002; 2004; Crandall et al. 2001]. The devices mostly used are monotonicity, the Harnack inequality, comparison, cone comparison and the boundary Harnack inequality for flat boundaries. For discussion see [Bhattacharya 2002; Crandall et al. 2001]. Also see [Aronsson et al. 2004; Bhattacharya et al. 1989] for more information of the origins of such questions and issues related to $\infty$-harmonic functions. 
We have divided our work as follows. In Section 2, we introduce notations needed in our work, and recall some preliminary results about $\infty$-harmonic functions. We prove Theorem 1.1 in Section 3, and the proof of Theorem 1.2 appears in Section 4. Finally, in Section 5, we present a short discussion in the case of the infinite strip.

\section{Notations and Preliminaries}

Let $O$ be the origin in $\mathbb{R}^{n}$, let $\bar{U}$ denote the closure of a set $U$ in $\mathbb{R}^{n}$, and let $\vec{e}_{n}$ be the unit vector parallel to the positive $x_{n}$-axis. Let $B(r, P)$ denote the open ball in $\mathbb{R}^{n}$ with center $P$ and radius $r>0$, let $\Omega(r, P)$ denote the intersection $\Omega \cap B(r, P)$, let $\partial(r, P)$ be $\partial \Omega \cap \bar{B}(r, P)$ and let $E(r, P)$ be $\partial B(r, P) \cap \bar{\Omega}$. For $A=\left(A_{1}, A_{2}, A_{3}, \ldots, A_{n}\right) \in \mathbb{R}^{n}$, let $x_{n}(A)=A_{n}$, let $A^{\prime}=\left(A_{1}, A_{2}, A_{3}, \ldots, A_{n-1}\right)$, let

$$
|P-Q|_{n-1}=\sqrt{\sum_{i=1}^{n-1}\left(P_{i}-Q_{i}\right)^{2}}
$$

and, for $t \in \mathbb{R}$, let $A+t \vec{e}_{n}=\left(A^{\prime}, A_{n}+t\right)$. For $P \in \mathbb{R}^{n}$, let $C(r, P)$ denote the cylinder $\left\{x \in \mathbb{R}^{n}: P_{n}<x_{n}<P_{n}+2 r,|x-P|_{n-1}<r\right\}$. Thus $C(r, P)$ has length $2 r$ and radius $r$, and its axis is parallel to the $x_{n}$-axis. Let $F(r, P)$ denote the flat face $\left\{x \in \mathbb{R}^{n}: x_{n}=P_{n},|x-P|_{n-1}<r\right\}$ of $C(r, P)$ which lies in the hyperplane $x_{n}=P_{n}$. We study the problem

$$
\begin{aligned}
\Delta_{\infty} u(x)=0 & \text { for } x \in \Omega, \\
u(x)=0 & \text { for } x \in \partial \Omega .
\end{aligned}
$$

We assume that $u(x) \geq 0$ for $x \in \Omega$ unless otherwise stated, and that $\partial \Omega$ will be smooth and $u$ continuous up to $\partial \Omega$. It is well known that $u$ is locally Lipschitz continuous in $\Omega$ (see [Bhattacharya 2002; Crandall et al. 2001; Jensen 1993; Lindqvist and Manfredi 1995]) and has the cone comparison property, and we make considerable use of these facts throughout this work. We now list a set of facts about $\infty$-harmonic functions.

We use the following version of the Harnack inequality [Bhattacharya 2002; 2004; Lindqvist and Manfredi 1995]: let $u>0$ be $\infty$-harmonic in $\Omega$, and let $\delta>0$ be such that the set $\Omega_{\delta}=\{x \in \Omega: \operatorname{dist}(x, \partial \Omega) \geq \delta\}$ is not empty. If $P$ and $Q$ are points in $\Omega_{\delta}$ and the segment $P Q \subset \Omega_{\delta}$, then $u(P) \geq e^{-|P-Q| / \delta} u(Q)$. If $P$ is joined to $Q$ by a smooth path in $\Omega_{\delta}$, with arc length $l(P, Q)$ then

$$
u(P) \geq e^{-l(P, Q) / \delta} u(Q) .
$$

Monotonicity plays a crucial role here [Bhattacharya 2002, Lemma 3.6; 2004, Lemma 3]. Let $u \geq 0$ be $\infty$-harmonic in $\Omega$, and $B(r, z) \subset \Omega$. For $x \in B(r, z)$, define $d(x)=r-|x-z|=\operatorname{dist}(x, \partial B(r, z))$; if $\vec{\eta}$ is a unit vector and $0 \leq s<t<r$, 
then

$$
\frac{u(z)}{r}=\frac{u(z)}{d(z)} \leq \frac{u(z+s \vec{\eta})}{d(z+s \vec{\eta})}=\frac{u(z+s \vec{\eta})}{r-s} \leq \frac{u(z+t \vec{\eta})}{r-t}=\frac{u(z+t \vec{\eta})}{d(z+t \vec{\eta})}
$$

We will need a different version of (2). We take $u=0$ on $\partial \Omega$, and for $z \in \mathbb{R}^{n}$ we define $M(r, z)=\sup _{\Omega(r, z)} u(x)=\sup _{\partial \Omega(r, z)} u(x)$.

Lemma 2.1. Let $u \geq 0$ be $\infty$-harmonic in $\Omega$ and $\left.u\right|_{\partial \Omega}=0$; suppose $z \in \mathbb{R}^{n}$, and $r>0$ is such that $\Omega(r, z)$ is not empty. Let $x, y \in \Omega(r, z)$ be on the same radial line through $z$, with $|x-z|<|y-z|<r$, and suppose that $u(x) \leq l+(M(r, z)-l)|x-z| / r$ for some $l \in \mathbb{R}$, and all $x \in \Omega(r, z)$. Then

$$
\frac{M(r, z)-l}{r} \leq \frac{M(r, z)-u(x)}{r-|x-z|} \leq \frac{M(r, z)-u(y)}{r-|y-z|} .
$$

If $z \in \bar{\Omega}$ this holds with $u(z)$ in place of $l$.

Proof. Fix $x \in \Omega$, set $B(r, x, z)=B(r-|x-z|, x)$ and $O(x, z, r)=B(r, x, z) \cap \Omega$. For $w \in O(x, z, r)$ define

$$
\omega(w)=u(x)+\frac{(M(r, z)-u(x))|w-x|}{r-|x-z|} .
$$

Then $u \leq \omega$ on $\partial B(r, x, z) \cap \bar{\Omega}$ and $\partial \Omega \cap \bar{B}(r, x, z)$, and $u(x)=\omega(x)$. By comparison, $u \leq \omega$ in $O(x, z, r)$ [Barles and Busca 2001; Bhattacharya 2002; Crandall et al. 2001; Jensen 1993]. Note that $O(x, z, r) \subset \Omega(r, z)$. The first inequality follows trivially, and the second follows by taking $w=y$. Let $z \in \bar{\Omega}$ and define

$$
v(x)=u(z)+\frac{(M(r, z)-u(z))|x-z|}{r}
$$

in $\Omega(r, z)$. By comparison, $u(x) \leq v(x)$ in $\Omega(r, z)$ and the claim follows.

We recall the boundary Harnack inequality [Bhattacharya 2002]. Let $P \in \mathbb{R}^{n}$ and $s>0$. Suppose that $u_{1}, u_{2}>0$ are $\infty$-harmonic in $C(8 s, P)$, and vanish continuously on $F(8 s, P)$. Then there exist constants $M_{1}$ and $M_{2}$, independent of $s$ and $u_{i}$, such that for all $x \in C(s, P)$,

$$
M_{1} \frac{u_{1}(z)}{u_{2}(z)} \leq \frac{u_{1}(x)}{u_{2}(x)} \leq M_{2} \frac{u_{1}(z)}{u_{2}(z)}
$$

where $z=\left(P^{\prime}, P_{n}+2 s\right)$. We now assume that $\Omega$ is unbounded and show that nonconstant $\infty$-harmonic functions, with unrestricted sign, have at least linear growth. If $u \geq 0$ and has linear growth in $\Omega$ then Lemma 2.3 implies global Lipschitz continuity.

Lemma 2.2. Let $u$ be $\infty$-harmonic in $\Omega$ such that $\left.u\right|_{\partial \Omega}=0$. Fix $z \in \mathbb{R}^{n}$ and $t \geq 0$, and define $\delta=\operatorname{dist}(z, \Omega)$. Then 
(i) $M(r, z)$ is convex in $r$, for all $r \geq \delta$, and

(ii) $M(r, z) \geq M(\delta, z)+(M(t, z)-M(\delta, z))\left(\frac{r-\delta}{t-\delta}\right)$ for $r>t>\delta$.

Proof. Set $\rho(x)=|x-z|$ and choose $a, b$ and $c$ such that $\delta<a<c<b$. As the intersection $\partial B(a, z) \cap \partial \Omega$ is not empty, by the maximum principle,

$$
0 \leq M(a, z) \leq M(c, z) \leq M(b, z) .
$$

Define $v(x)=M(a, z)+(M(b, z)-M(a, z))(\rho(x)-a) /(b-a) \geq 0$ for all $x$ in $\Omega(b, z) \backslash \bar{\Omega}(a, z)$. Clearly, $u \leq v$ on $\partial B(a, z) \cap \bar{\Omega}$ and on $\partial B(b, z) \cap \bar{\Omega}$, and $u=0 \leq v$ on $\partial \Omega \cap(\bar{B}(b, z) \backslash B(a, z))$. By the cone comparison, $u(x) \leq v(x)$ for all $x \in \Omega(b, z) \backslash \bar{\Omega}(a, z)$. Hence

$$
\sup _{|x-z|=c} u(x)=M(c, z) \leq M(a, z)+(M(b, z)-M(a, z))(c-a) /(b-a),
$$

and convexity follows. Since $(M(r, z)-M(a, z)) /(r-a)$ increases as $r$ increases, selecting $a=\delta$ and $r>t>\delta$, a simple rearrangement yields part (ii). Note that $M(\delta, z)=0$ if $z \in \mathbb{R}^{n} \backslash \bar{\Omega}$, and $M(\delta, z)=M(0, z)=u(z)$ if $z \in \bar{\Omega}$.

Lemma 2.3. Let $u \geq 0$ be $\infty$-harmonic in $\Omega$ and $\left.u\right|_{\partial \Omega}=0$. If for some $z \in \Omega$, some $C>0$ and some $a>0, M(r, z) \leq C r$ for all $r \geq a$, then $u$ is globally Lipschitz continuous in $\Omega$, with Lipschitz constant $C$.

Proof. Fix $x, y \in \Omega$. For $\rho>0$, let $v(w)=u(x)+(M(\rho, x)-u(x))(|w-x| / \rho)$ in $\Omega(\rho, x)$. Then $u \leq v=M(\rho, x)$, on $\partial B(\rho, x) \cap \Omega$. Also, $u=0 \leq v$ on $\partial \Omega \cap B(\rho, x)$ and $u(x)=v(x)$. By comparison, $u \leq v$ in $\Omega(\rho, x)$. By the maximum principle, $M(\rho, x) \leq M(\rho+|x-z|, z) \leq C(\rho+|x-z|)$. Set $w=y$ and $\rho>|x-y|$, then

$$
\begin{aligned}
\frac{u(y)-u(x)}{|x-y|} & \leq \frac{M(\rho, x)-u(x)}{\rho} \\
& \leq \frac{M(\rho+|x-z|, z)-u(x)}{\rho} \leq C\left(1+\frac{|x-z|}{\rho}-\frac{u(x)}{\rho}\right) .
\end{aligned}
$$

The claim follows by letting $\rho \rightarrow \infty$.

\section{The infinite half-space}

Here $\Omega \subset \mathbb{R}^{n}$ is the half-space $H=\left\{x \in \mathbb{R}^{n}: x_{n}>0\right\}$ and $H_{0}$ is the hyperplane $x_{n}=0$. Also $u \geq 0$ is $\infty$-harmonic in $H$ and vanishes continuously on $H_{0}$. By the Harnack inequality, $u>0$ in $H$. We will prove that $u(x)=C x_{n}$ in $H$ for some $C>0$. This will be the consequence of several lemmas. For $P \in \mathbb{R}^{n}$, it follows that $\partial(r, P)=\partial \Omega \cap \bar{B}(r, P)=H_{0} \cap \bar{B}(r, P)$ and $E(r, P)=\partial B(r, P) \cap \bar{\Omega}=\partial B(r, P) \cap \bar{H}$. Thus $\partial \Omega(r, P)=\partial(r, P) \cup E(r, P)$. If $\Omega(r, P)$ is not empty, then by the maximum 
principle

$$
M(r, P)=\sup _{\Omega(r, P)} u(x)=\sup _{\partial \Omega(r, P)} u(x)=\sup _{E(r, P)} u(x)>0 .
$$

If $P \in H$, then $M(0, P)=u(P)$. We introduce some additional notation. For $S \in H_{0}$, let

$$
T(S)=\left\{x: x=\left(S_{1}, S_{2}, \ldots, S_{n-1}, t\right)=\left(S^{\prime}, t\right) \text { for } t \geq 0\right\}
$$

be the straight ray in $H$, starting at $S$ and parallel to the $x_{n}$-axis. Set $u(S+t)=$ $u\left(S+t \vec{e}_{n}\right)$ for $t>0$, and let $B(\theta, S+\theta)$ be the ball of radius $\theta>0$ centered at $S+\theta \vec{e}_{n}$.

Lemma 3.1. Let $u>0$ be $\infty$-harmonic in $H$ such that $\left.u\right|_{H_{0}}=0$, and let $x_{n}>0$. Then for every $S \in H_{0}$

(i) $u\left(S+x_{n}\right) / x_{n}$ is decreasing in $x_{n}$ and $\lim _{x_{n} \uparrow \infty} u\left(S+x_{n}\right) / x_{n}=L(S, \infty)<\infty$,

(ii) $0<\lim _{x_{n} \downarrow 0} u\left(S+x_{n}\right) / x_{n}=L(S, 0)<\infty$, and

(iii) $0<L(S, \infty) \leq L(S, 0)$.

Moreover, there is a positive number $L$ such that $L(S, \infty)=L$ for all $S \in H_{0}$.

Proof. Let $S \in H_{0}$ and, for $x_{n}>0$, consider the ball $B=B\left(x_{n}, S+x_{n}\right)$. If $0<y_{n}<x_{n}$ then $S+y_{n}$ and $S+x_{n}$ lie in $T(S)$. Also $y_{n}=\operatorname{dist}\left(S+y_{n}, \partial B\right)$ and $x_{n}=\operatorname{dist}\left(x_{n}, \partial B\right)$. Monotonicity (2) implies that $u\left(S+x_{n}\right) / x_{n} \leq u\left(S+y_{n}\right) / y_{n}$. Thus the first assertion follows and implies the second. Except for the finiteness of $L(S, 0)$, the third assertion follows from the first two. To show that $L(S, 0)$ is finite, consider the function $v(x)=M(1, S)|x-S|$ in $\Omega(1, S)$. Clearly $v(x) \geq u(x)$ on $\partial \Omega(1, S)$. By comparison, $u(x) \leq v(x)$ in $\Omega(1, S)$ and $u\left(S+x_{n}\right) \leq M(1, S) x_{n}$ for $0 \leq x_{n} \leq 1$. Thus $0<L(S, 0) \leq M(1, S)<\infty$. We now show that the $L(S, \infty)$ are all equal. Take $x_{n}>|S|$, then $S+x_{n} \in B\left(x_{n}, O+x_{n}\right)$ and $\operatorname{dist}\left(S+x_{n}, \partial B\left(x_{n}, O+x_{n}\right)\right)=x_{n}-|S|$. By (2), $u\left(O+x_{n}\right) / x_{n} \leq u\left(S+x_{n}\right) /\left(x_{n}-|S|\right)$. Then $L(O, \infty) \leq L(S, \infty)$ by letting $x_{n} \rightarrow \infty$. Switch $S$ with $O$ to get equality. We employ the boundary Harnack inequality (3) to show that $L>0$. We select $u_{1}(x)=u(x)$ and $u_{2}(x)=x_{n}$. For all $s>0$, the cylinder $C(8 s, O)$ is contained in $H$; (3) then implies that

$$
M_{1} \frac{u\left(O+2 s \vec{e}_{n}\right)}{2 s} \leq \frac{u(x)}{x_{n}} \leq M_{2} \frac{u\left(O+2 s \vec{e}_{n}\right)}{2 s} \text { for all } x \in C(s, O) .
$$

Take $s>0$ large and fix $t \in(0, s)$. The preceding inequalities yield, for $x=O+t \vec{e}_{n}$,

$$
M_{1} L=\lim _{s \rightarrow \infty} M_{1} \frac{u\left(O+2 s \vec{e}_{n}\right)}{2 s} \leq \frac{u\left(O+t \vec{e}_{n}\right)}{t} \leq \lim _{s \rightarrow \infty} M_{2} \frac{u\left(O+2 s \vec{e}_{n}\right)}{2 s}=M_{2} L .
$$

Letting $t \rightarrow 0$, it follows $M_{1} L \leq L(O, 0) \leq M_{2} L$. It is clear that this estimate holds for every $S \in H$. 
Remark 3.2. Lemma 3.1 implies that $M_{1} L \leq b=\sup _{S \in H_{0}} L(S, 0) \leq M_{2} L<\infty$. By the first part of Lemma 3.1, we have $0<L x_{n} \leq u(x) \leq b x_{n}$ for $x \in H$.

This remark and Lemma 2.3 imply that $u$ is globally Lipschitz continuous in $H$. Thus there exists $K>0$, independent of $x, y \in H$, such that

$$
|u(x)-u(y)| \leq K|x-y| \text { for } x, y \in H .
$$

We now study $u$ on infinite strips in $H$. For $a>0$, define $H_{a}=\left\{x: x_{n}=a\right\}$, $H(a)=\left\{x: 0<x_{n}<a\right\}$, and $\partial H(a)=H_{0} \cup H_{a}$. Define

$$
\mu(a)=\sup _{H_{a}} u(x)>0 \quad \text { and } \quad F(a)=\sup _{H(a)} u(x)>0 .
$$

By Remark 3.2 and (5), $\mu(a)$ is bounded, and $F(a)$ is bounded and increasing.

Lemma 3.3. Let $u>0$ be as in Lemma 3.1. If $\mu(a)$ and $F(a)$ are as defined in (6),

$$
\mu(a)=F(a) \quad \text { and } \quad \mu(a)=\Lambda a,
$$

where $\Lambda=\mu(1)$ and $a>0$ is arbitrary.

Proof. By the Harnack inequality (1), $F(a)$ cannot be attained in the interior of $H(a)$. If $F(a)>\mu(a)$, then there is a sequence $\left\{P_{m}\right\}_{m=1}^{\infty}$ such that $0<x_{n}\left(P_{m}\right)<a$ for all $m,\left|P_{m}\right| \rightarrow \infty$ and $u\left(P_{m}\right) \rightarrow F(a)>\mu(a)$. We argue by contradiction. For each $m$, let $Q_{m}=\left(P_{m}^{\prime}, a\right) \in H_{a}$, then $u\left(Q_{m}\right) \leq \mu(a)$. By (5), we see that $u\left(P_{m}\right)-\mu(a) \leq u\left(P_{m}\right)-u\left(Q_{m}\right) \leq K\left(a-x_{n}\left(P_{m}\right)\right)$ for all $m$. Thus, for large $m$,

$$
a-x_{n}\left(P_{m}\right) \geq \frac{u\left(P_{m}\right)-u\left(Q_{m}\right)}{K} \geq \frac{3}{4} \frac{F(a)-\mu(a)}{K}=A>0 .
$$

For $0<\theta<1$, let $R_{m}(\theta)=\left(P_{m}^{\prime}, a-\theta A\right) \in H_{a-\theta A} \subset H(a)$. From (5), we see that $u\left(P_{m}\right) \leq K x_{n}\left(P_{m}\right)$ and so, for all $m$,

$$
\frac{F(a)}{K} \leq \liminf _{k \rightarrow \infty} x_{n}\left(P_{k}\right) \leq \limsup _{k \rightarrow \infty} x_{n}\left(P_{k}\right) \leq a-A<x_{n}\left(R_{m}(\theta)\right)=a-\theta A .
$$

Fix $\theta$; then (7) and (8) imply that, for large $m$,

$$
0<x_{n}\left(P_{m}\right) \leq a-A<x_{n}\left(R_{m}(\theta)\right)=a-\theta A,
$$

$$
\limsup _{k \rightarrow \infty}\left|P_{k}-R_{k}(\theta)\right|=\limsup _{k \rightarrow \infty}\left(x_{n}\left(R_{k}(\theta)\right)-x_{n}\left(P_{k}\right)\right) \leq(a-\theta A)-F(a) / K,
$$

and

$$
\min \left(\operatorname{dist}\left(P_{m}, \partial H(a)\right), \operatorname{dist}\left(R_{m}(\theta), \partial H(a)\right)\right) \geq \min (\theta A, F(a) / K)=B>0 .
$$


By applying the Harnack inequality to the function $v(x)=F(a)-u(x)$, we now see that

$$
\begin{aligned}
0 & =\lim _{m \rightarrow \infty}\left(F(a)-u\left(P_{m}\right)\right) \\
& \geq \exp \left(\frac{-((a-\theta A)-F(a) / K)}{B}\right) \lim _{m \rightarrow \infty}\left(F(a)-u\left(R_{m}(\theta)\right)\right) \geq 0 .
\end{aligned}
$$

Thus $u\left(R_{m}(\theta)\right) \rightarrow F(a)$ as $m \rightarrow \infty$. We show that this, together with (5), leads to a contradiction. Let $X_{m}(\theta)=\left(R_{m}(\theta)^{\prime}, a\right)$; then (7) holds. That is, for large $m$,

$$
\theta A=a-x_{n}\left(R_{m}(\theta)\right) \geq \frac{u\left(R_{m}(\theta)\right)-u\left(X_{m}(\theta)\right)}{K} \geq \frac{3}{4} \frac{F(a)-\mu(a)}{K}=A>0 .
$$

Thus $F(a)=\mu(a)$.

We now prove that $\mu(a)=\Lambda a$. Let $b>a$; we show that $\mu(a) / a \geq \mu(b) / b$. By the first part of Lemma 3.1, $u\left(S+a \vec{e}_{n}\right) / a \geq u\left(S+b \vec{e}_{n}\right) / b$ for all $S \in H_{0}$. Now take the supremum of both sides. We claim that $\mu(a)$ is convex in $a$. Let $S \in H_{0}$ and, for $0 \leq s<t$, set $r=(s+t) / 2$. Consider

$$
v_{s, t}(x)=\mu(s)+(\mu(t)-\mu(s)) \frac{|x-S|-s}{t-s} \geq 0,
$$

for all $x \in \Omega(t, S) \backslash \bar{\Omega}(s, S)$. Using the equality $F(a)=\mu(a)$ and the cone comparison we see that $u \leq v_{s, t}$ in $\Omega(t, S) \backslash \bar{\Omega}(s, S)$. Now we take $x=S+r \vec{e}_{n}$ to see that $\mu(r)=\sup _{S \in H_{0}} u\left(S+r \vec{e}_{n}\right) \leq \frac{1}{2}(\mu(s)+\mu(t))$. Convexity follows. Since $\mu(0)=0$, we see that $\mu(a) / a$ is both increasing and decreasing as a function of $a-$ in other words, it is constant.

Proof of Theorem 1.1. It is clear that Theorem 1.1 would follow if we could show that $\Lambda=L$. For $Q \in H_{0}$ and $r>0$, set $P_{Q}(r)=\left(Q^{\prime}, r\right)$. For $0 \leq \varepsilon<\Lambda$, let $Q=Q(\varepsilon) \in H_{0}$ be such that $u\left(P_{Q(\varepsilon)}(1)\right) \geq \Lambda-\varepsilon=\mu(1)-\varepsilon$. We fix $\varepsilon$ and $Q$, and suppress the argument $\varepsilon$. By Lemma 3.1(i) we have $u\left(P_{Q}(1)\right) \leq u\left(Q+x_{n}\right) / x_{n}$ for $0<x_{n}<1$. Thus

$$
u\left(P_{Q}\left(x_{n}\right)\right)=u\left(Q+x_{n}\right) \geq(\Lambda-\varepsilon) x_{n} \quad \text { for } 0<x_{n} \leq 1 .
$$

Since $M(0, Q)=0$, Lemma 2.2(i) and equation (4) imply that $M(r, Q) / r$ is increasing. From (9)

$$
M(1, Q) \geq u\left(P_{Q}(1)\right) \geq \Lambda-\varepsilon \quad \text { and } \quad M(r, Q) \geq(\Lambda-\varepsilon) r \quad \text { for } r \geq 1 .
$$

For $r>0$, define $t=t(\varepsilon, r)$ by $\mu(t)=\Lambda t=(\Lambda-\varepsilon) r$. Let $T=T(r, \varepsilon) \in \partial B(r, Q)$ be such that $u(T)=M(r, Q)$. By Lemma 3.3 and (10), $u(x)<\mu(t)$ for all $x \in H(t)$; moreover

$$
T \in \partial B(r, Q) \cap\left\{x: x_{n} \geq t\right\} \quad \text { and } \quad x_{n}(T) \geq t=\left(1-\frac{\varepsilon}{\Lambda}\right) r .
$$


Let $\Upsilon=\Upsilon(\varepsilon, Q)$ be the interior of the cone with vertex $Q$, axis parallel to $\vec{e}_{n}$ and half-angle $\theta=\theta(\varepsilon, Q)=\cos ^{-1}(1-\varepsilon / \Lambda)$. Clearly (11) implies that $T$ lies in the intersection $\bar{\Upsilon} \cap \partial B(r, Q)$. Since the points $P_{Q}(r)$ and $T$ lie on $\partial B(r, Q)$, the arc length of $P_{Q}(r) T$, along a great circle, is at most $\theta r$. The distance to $H_{0}$ is at least $t$. Applying the Harnack inequality (1) to $u\left(P_{Q}(r)\right)$ and $u(T)=M(r, Q)$, and using (10), we see that for $r>1$,

$$
u\left(P_{Q}(r)\right) \geq \exp \left(-\frac{\theta}{1-\varepsilon / \Lambda}\right) M(r, Q) \geq \exp \left(-\frac{\cos ^{-1}(1-\varepsilon / \Lambda)}{1-\varepsilon / \Lambda}\right)(\Lambda-\varepsilon) r,
$$

and so

$$
L=\lim _{r \uparrow \infty} \frac{u\left(Q+r \vec{e}_{n}\right)}{r}=\lim _{r \uparrow \infty} \frac{u\left(P_{Q}(r)\right)}{r} \geq \exp \left(-\frac{\cos ^{-1}(1-\varepsilon / \Lambda)}{1-\varepsilon / \Lambda}\right)(\Lambda-\varepsilon) .
$$

Since this holds for all $\varepsilon>0$, it follows that $L \geq \Lambda$ and $u(x)=\Lambda x_{n}$ for $x_{n}>0$.

\section{The exterior of a ball}

Let $\Omega=\mathbb{R}^{n} \backslash \bar{B}(1, O)$, and assume that $u>0$ and $\left.u\right|_{\partial \Omega}=0$. We prove that $u=K(|x|-1)$ for some $K>0$. For $r>1$ set

$$
\begin{aligned}
& \mu(r)=\sup _{|x|=r} u(x)=\sup _{B(r, O) \backslash B(1, O)} u(x), \\
& m(r)=\inf _{|x|=r} u(x)>0 .
\end{aligned}
$$

(On the first line we have used the maximum principle.) Clearly, $\mu(1)=m(1)=0$. Let $S^{n-1}$ be the unit sphere in $\mathbb{R}^{n}$, and for $t>1$ and $\omega \in S^{n-1}$ set $\Delta(t, \omega)=$ $u(t \omega) /(t-1)$.

Lemma 4.1. Let $u>0$ be $\infty$-harmonic in $\mathbb{R}^{n} \backslash B(1, O)$ and $\left.u\right|_{\partial B(1, O)}=0$. Let $\mu$, $m$ and $\Delta$ be as defined above. Then

(i) $u(t \omega) /(t-1)$ decreases as $t$ increases, and $\lim _{t \rightarrow \infty} u(t \omega) /(t-1)=L(\omega)>0$ for all $\omega \in S^{n-1}$;

(ii) $\mu(2)=\mu(t) /(t-1)$ for all $t>1$, and $L(\omega) \leq \mu(2)$ for all $\omega \in S^{n-1}$;

(iii) $m(t) /(t-1)$ decreases as $t$ increases, and $L(\omega) \geq \lim _{t \rightarrow \infty} m(t) /(t-1) \geq$ $e^{-\pi} \mu(2)>0$, for all $\omega \in S^{n-1}$;

(iv) there exists $K>0$ such that, if $\min \left(t_{1}, t_{2}\right)>4, \max \left(t_{1}, t_{2}\right)<10 \min \left(t_{1}, t_{2}\right)$, and $\alpha=\cos ^{-1}\left\langle\omega_{1}, \omega_{2}\right\rangle$ for $\omega_{1}, \omega_{2} \in S^{n-1}$, then

$$
\left|\Delta\left(t_{1}, \omega_{1}\right)-\Delta\left(t_{2}, \omega_{2}\right)\right| \leq K\left(\frac{\left|t_{1}-t_{2}\right|}{t_{2}-1}+\alpha\right) .
$$

Proof. Parts (i), (ii) and (iii) follow from (2) and are interrelated. Fix $\omega \in S^{n-1}$; for $t>1$, consider the ball $B(t-1, t \omega)$. If $1<s<t$ then $\operatorname{dist}(s \omega, \partial B(t-1, t \omega))=s-1$, 
and (2) implies $u(s \omega) /(s-1) \geq u(t \omega) /(t-1)$. Thus $L(\omega) \geq 0$ exists (for positivity see part (iii)). Taking the supremum over $\omega$ on both sides, we see that $\mu(t) /(t-1)$ decreases as $t$ increases. Similarly, $m(t) /(t-1)$ decreases as $t$ increases. By Lemma 2.2, $\mu(t)$ is convex in $t \geq 1$ and since $\mu(1)=0, \mu(t) /(t-1)$ increases as $t$ increases. Thus $\mu(t)$ is linear in $t-1$ and part (ii) follows. Let $P(t), Q(t) \in$ $\partial B(t, O)$ be such that $u(P(t))=\mu(t)$ and $u(Q(t))=m(t)$. The arc length of $P(t) Q(t)$ along a great circle does not exceed $\pi t$, and $\operatorname{dist}(P(t) Q(t), \partial B(1, O))=$ $t-1$. Applying the Harnack inequality (1),

$$
\frac{u(t \omega)}{t-1} \geq \frac{m(t)}{t-1}=\frac{u(Q(t))}{t-1} \geq \frac{u(P(t))}{t-1} \exp \left(\frac{-\pi t}{t-1}\right)=\mu(2) \exp \left(\frac{-\pi t}{t-1}\right) .
$$

Part (iii) follows by letting $t \rightarrow \infty$. To see (iv), fix $\omega_{1}$ and $\omega_{2}$ in $S^{n-1}$ and let $0 \leq \alpha=\cos ^{-1}\left\langle\omega_{1}, \omega_{2}\right\rangle \leq \pi$. Take $\min \left(t_{1}, t_{2}\right)>4$ and $t_{1} \leq t_{2} \leq 10 t_{1}$. The distance from $t_{1} \omega_{1}$ to $t_{2} \omega_{2}$ is estimated by going from $t_{1} \omega_{1}$ to $t_{1} \omega_{2}$ along a great circle, and then from $t_{1} \omega_{2}$ to $t_{2} \omega_{2}$. Setting $\delta=\left|t_{1}-t_{2}\right|+t_{1} \alpha$ and $d=t_{1}-1$ (the distance to the boundary), the Harnack inequality implies $u\left(t_{1} \omega_{1}\right) \leq e^{\delta / d} u\left(t_{2} \omega_{2}\right)$. Set $J=$ $\max \left(u\left(t_{1} \omega_{1}\right), u\left(t_{2} \omega_{2}\right)\right)$; then, for some $K_{1}=K_{1}(\mu(2))>0$ and $K=K(\mu(2))>0$,

$$
\begin{aligned}
\Delta\left(t_{1}, \omega_{1}\right)-\Delta\left(t_{2}, \omega_{2}\right) & =\frac{u\left(t_{1} \omega_{1}\right)\left(t_{2}-t_{1}\right)}{\left(t_{1}-1\right)\left(t_{2}-1\right)}+\frac{u\left(t_{1} \omega_{1}\right)-u\left(t_{2} \omega_{2}\right)}{t_{2}-1} \\
& \leq \mu(2) \frac{\left|t_{2}-t_{1}\right|}{t_{2}-1}+J \frac{e^{\delta / d}-1}{t_{2}-1} \\
& \leq K_{1}\left(\frac{\left|t_{2}-t_{1}\right|}{t_{2}-1}+\frac{\delta}{d}\right) \leq K\left(\frac{\left|t_{2}-t_{1}\right|}{t_{2}-1}+\alpha\right),
\end{aligned}
$$

which proves part (iv).

Remark 4.2. From Lemma 4.1, if $L=\inf _{\omega \in S^{n-1}} L(\omega)$, then $L \leq u(x) /(|x|-1) \leq$ $\mu(2)$ for all $x \in \Omega$. Also $\left|L\left(\omega_{1}\right)-L\left(\omega_{2}\right)\right| \leq \mu(2)\left(e^{\alpha}-1\right) \leq C\left|\omega_{1}-\omega_{2}\right|$.

Remark 4.3. As in Section 3, there is a ray through $O$ on which $\mu(t)$ is attained for every $t>1$. To see this, let $P(t) \in \partial B(t, O)$ be such that $u(P(t))=\mu(t)$, and let $\omega(t)=P(t) /|P(t)|$. Since $S^{n-1}$ is compact, there is a sequence $\left\{t_{m}\right\}_{m=1}^{\infty}$ so that $t_{m} \uparrow \infty, \omega\left(t_{m}\right) \rightarrow \omega_{0}$, and $\theta_{m}=\cos ^{-1}\left\langle\omega\left(t_{m}\right), \omega_{0}\right\rangle \rightarrow 0$ as $m \uparrow \infty$. Setting $Q_{m}=t_{m} \omega_{0}$, the Harnack inequality (1) and Lemma 4.1 imply that

$$
\mu(2)=\frac{u\left(P\left(t_{m}\right)\right)}{t_{m}-1} \geq \frac{u\left(Q_{m}\right)}{t_{m}-1} \geq \frac{u\left(P\left(t_{m}\right)\right)}{t_{m}-1} \exp \left(\frac{-\theta_{m} t_{m}}{t_{m}-1}\right)=\mu(2) \exp \left(\frac{-\theta_{m} t_{m}}{t_{m}-1}\right)
$$

for all $m$. Clearly $L\left(\omega_{0}\right)=\mu(2)$ and the claim follows.

We now prove that $u(t \omega)=L(\omega)(t-1)$ for all $\omega \in S^{n-1}$ and all $t>1$ (see Lemma 4.5). This depends on a comparison result, Lemma 4.4, involving $u$ and a scaled version of $u$, and uses the fact that the $\Delta(t, \omega)$, for different values of 
$\omega$, are comparable at large values of $t$. Now some notation for Lemma 4.4: fix $P \in \partial B(1, O)$. For $\omega \in S^{n-1}$, let $R_{1}=R_{1}(\omega)$ be the ray $\{O+s \omega, s \geq 0\}$, and $R_{2}=R_{2}(\omega)$ be the ray $\{P+s \omega, s \geq 0\}$; also set $Q=Q(\omega)=O+\omega \in \partial B(1, O)$. For $x \in \Omega$, let $\omega=\omega(x)=(x-P) /|x-P|$, then $x=P+|x-P| \omega(x) \in R_{2}(w(x))$. We define $y=y(x, P)=Q(\omega(x))+(x-P)$, so $|y-Q|=|x-P|$. We scale $x$ as follows: for $\theta>1$, set $x_{\theta}=x_{\theta}(P)=P+\theta(x-P)$ and $y_{\theta}=y_{\theta}(x, P)=Q+\theta(x-P)$. Then $y, y_{\theta} \in R_{1}(\omega(x))$ and $x, x_{\theta} \in R_{2}(\omega(x))$, and $y-x=y_{\theta}-x_{\theta}=Q-P$; thus $|y-x|=\left|y_{\theta}-x_{\theta}\right| \leq 2$. Now set $u_{\theta}(x)=u_{\theta}(x, P)=u\left(x_{\theta}\right)=u(P+\theta(x-P))$. Clearly for fixed $P$ and $\theta>0, u_{\theta}(x)$ is $\infty$-harmonic.

Lemma 4.4. Let $u>0$ be as in Lemma 4.1 and $P \in \partial B(1, O)$. For $\theta>1$ and $x \in \Omega$, let $\omega(x), R_{1}, R_{2}, Q, x_{\theta}$, and $y_{\theta}$ be as defined above. Set $u_{\theta}(x)=u_{\theta}(x, P)=u\left(x_{\theta}\right)$; if $1<s<\theta$, then $u_{\theta}(x) \geq s u(x)$ for all $x \in \Omega$. Then $u_{\theta}(x) \geq \theta u(x)$ for all $x \in \Omega$.

Proof. This is done in four steps. Fix $P$ and $\theta>1$. We show that there exists $\rho>1$ such that $u_{\theta}(x) \geq s u(x)$ for all $x \in \partial B(r, O)$ and $r \geq \rho$. Comparison will then imply the lemma.

Step 1: Properties of $u_{\theta}$. Clearly the set

$$
Z_{\theta}=\left\{x: u_{\theta}(x)=0\right\}=\{|x-(1-1 / \theta) P|=1 / \theta\}
$$

lies in $\bar{B}(1, O)$. Thus $u_{\theta}(x) \geq 0$ on $\partial B(1, O)$. Since $|Q|=|P|=1$, we have

(a) $\theta|x-P|-1 \leq\left|x_{\theta}\right| \leq \theta|x-P|+1$,

(b) $\left|y_{\theta}\right|=\theta|x-P|+1$, and

(c) $|x-P|-1 \leq|x| \leq|x-P|+1$.

Thus $\operatorname{dist}\left(y_{\theta}, \partial B(1, O)\right)=\left|y_{\theta}\right|-1 \approx \operatorname{dist}\left(x_{\theta}, \partial B(1, O)\right)=\left|x_{\theta}\right|-1$ when $|x|$ is large. From (a), (b) and the Harnack inequality,

$$
u\left(y_{\theta}\right) \exp \left(-\frac{\left|y_{\theta}-x_{\theta}\right|}{\theta|x-P|}\right) \leq u_{\theta}(x)=u\left(x_{\theta}\right) \leq u\left(y_{\theta}\right) \exp \left(\frac{\left|y_{\theta}-x_{\theta}\right|}{\theta|x-P|-2}\right) .
$$

Fix $\omega$ and select $x, x_{\theta} \in R_{2}(\omega)$, and $y, y_{\theta} \in R_{1}(\omega)$. Divide by $\left|y_{\theta}\right|-1$ and note that (a), (b), (c) and Lemma 4.1 imply that

$$
\lim _{x \rightarrow \infty} \frac{u_{\theta}(x)}{|x|-1}=\theta L(\omega) \text { and } \lim _{x \rightarrow \infty} \frac{u(x)}{|x|-1}=L(\omega),
$$

since $\left|y_{\theta}-x_{\theta}\right|=|P-Q|$. The second conclusion follows by working similarly with $u, x$ and $y$.

Step 2. Fix $1<s<\theta$ and let $\varepsilon_{1}>0$ and $\varepsilon_{2}>0$ be such that $\varepsilon_{1}+\varepsilon_{2}<L(\theta-s)$, where $L=\inf _{\omega} L(\omega)$. Then $\theta L(\omega)-\varepsilon_{1}>s L(\omega)+\varepsilon_{2}$ for all $\omega \in S^{n-1}$. From (12) 
there is a $\rho=\rho\left(\varepsilon_{1}, \varepsilon_{2}, s, \omega\right)>0$, such that for all $x \in R_{2}(\omega)$ with $|x| \geq \rho$,

$$
\frac{u_{\theta}(x)}{|x|-1}>\theta L(\omega)-\varepsilon_{1}>s L(\omega)+\varepsilon_{2}>\frac{s u(x)}{|x|-1} .
$$

In Step 3, we show that there is a $\rho$ such that (13) holds independently of the choice of $\omega \in S^{n-1}$.

Step 3. We first show that there is a $\rho>0$ such that the first inequality in (13) holds for all $\omega$. Recall that $\omega=\omega(v)=(v-P) /|v-P|$ for $v \in \partial B(r, O)$. We prove that the quantity

$$
D\left(\theta, r, v, \omega, \varepsilon_{1}\right)=\frac{u_{\theta}(v)}{|v|-1}-\left(\theta L(\omega)-\varepsilon_{1}\right)
$$

is continuous in $\omega$ and positive for large $r$, for all $v \in \partial B(r, O)$. Let $\omega_{1} \in S^{n-1}$ and let $x$ lie in $R_{2}\left(\omega_{1}\right) \cap \partial B(r, O)$, with $r>20$; take $\omega_{2}$ close to $\omega_{1}$ and let $z$ lie in $R_{2}\left(\omega_{2}\right) \cap \partial B(r, O)$. By Remark 4.2, $\theta\left|L\left(\omega_{2}\right)-L\left(\omega_{1}\right)\right|$ is small. Clearly, $\max (|z-P|,|x-P|) \leq r+1$. Noting that $z_{\theta}=P+\theta(z-P)$ and $x_{\theta}=P+\theta(x-P)$, we see that $\left|z_{\theta}-x_{\theta}\right|=\theta|x-z| \leq \theta(r+5) \alpha$, where $\alpha=\cos ^{-1}\left\langle\omega_{1}, \omega_{2}\right\rangle$. From (a) and (c) in Step 1, we see that, for large $r$,

$$
\theta(r-1) \approx \max \left(\left|x_{\theta}\right|-1,\left|z_{\theta}\right|-1\right) \geq \min \left(\left|x_{\theta}\right|-1,\left|z_{\theta}\right|-1\right) \approx \theta(r-1) .
$$

By the Harnack inequality, $u_{\theta}(z)=u\left(z_{\theta}\right) \geq u_{\theta}(x) \exp (-\alpha(r+5) /(r-1))$. Thus $u_{\theta}(x) \leq u_{\theta}(z) e^{25 \alpha / 19}$. By Remark 4.2, $u_{\theta}(v)=u\left(v_{\theta}\right) \leq \mu(2)\left(\left|v_{\theta}\right|-1\right)$. Thus (12) yields

$$
\frac{\left|u_{\theta}(x)-u_{\theta}(z)\right|}{|x|-1} \leq \frac{\sup \left(u_{\theta}(x), u_{\theta}(z)\right)}{|x|-1}\left(e^{25 \alpha / 19}-1\right) \leq \theta \mu(2)\left(e^{25 \alpha / 19}-1\right) .
$$

This and Remark 4.2 yield, for some $C>0$ independent of $r$,

$$
\left|D\left(\theta, r, x, \omega_{1}, \varepsilon_{1}\right)-D\left(\theta, r, z, \omega_{2}, \varepsilon_{1}\right)\right| \leq C \theta \alpha
$$

for $x, z \in \partial B(r, O)$ and $r>20$. Fix $\omega_{1}$, and let $\rho$ be such that $D\left(\theta, r, x, \omega_{1}, \varepsilon_{1}\right)>$ $\frac{1}{2} \varepsilon_{1}$ for all $r \geq \rho$ and $x \in R_{2}\left(\omega_{1}\right) \cap \partial B(r, O)$. By (15), in a fixed small $\omega$ neighborhood, positivity of $D$ persists. The conclusion follows by the compactness of $S^{n-1}$.

We now discuss the second inequality in (12). Let $x \in R_{2}\left(\omega_{1}\right) \cap \partial B(r, O)$ and $z \in R_{2}\left(\omega_{2}\right) \cap \partial B(r, O)$ with $\alpha$ small. Clearly

$$
|u(x)-u(z)| \leq \max (u(x), u(z)) e^{(r+5) \alpha /(\mid r-1)} .
$$


Applying Remark 4.2, Lemma 4.1 and selecting $r \geq \rho$ to be large, we see that

$$
\begin{aligned}
& \left|\left(s L\left(\omega_{1}\right)+\varepsilon_{2}-\frac{s u(x)}{|x|-1}\right)-\left(s L\left(\omega_{2}\right)+\varepsilon_{2}-\frac{s u(z)}{|z|-1}\right)\right| \\
& \quad \leq s \frac{|u(x)-u(z)|}{|x|-1}+s\left|L\left(\omega_{1}\right)-L\left(\omega_{2}\right)\right| \leq s \mu(2)\left(e^{2 \alpha}-1\right)+K s \alpha .
\end{aligned}
$$

We again use the compactness of $S^{n-1}$.

Step 4. From Step $1, u_{\theta} \geq 0=s u$ on $\partial B(1, O)$. From Step 3, with $\varepsilon_{1}$ and $\varepsilon_{2}$ as in Step 2, and for all $r \geq \rho\left(\varepsilon_{1}, \varepsilon_{2}\right)$, we see that $u_{\theta}>s u$ in $\partial B(r, O)$. By comparison, $u_{\theta} \geq s u$ in $B(\rho, O) \backslash \bar{B}(1, O)$. This holds in all of $\Omega$, and for all $1<s<\theta$. Thus $u_{\theta}(x) \geq \theta u(x)$ for all $x \in \Omega$.

Next we show that Lemma 4.4 implies that $u$ is linear along rays through $O$.

Lemma 4.5. For every $\omega \in S^{n-1}$, let $T(\omega)$ be the ray $\{O+s \omega: s \geq 0\}$, and let $P=$ $O+\omega$. Let $\theta>1$, and let $u$ and $u_{\theta}$ be as in Lemma 4.4. Then $u(x)=L(\omega)(|x|-1)$ for all $x \in T(\omega) \cap \Omega$.

Proof. Fix $x, y \in T(\omega) \cap \Omega$ with $|x-P|<|y-P|$, and define $\theta=|y-P| /|x-P|$. Then $y=P+\theta(x-P)=x_{\theta}$, and so $u_{\theta}(x)=u\left(x_{\theta}\right)=u(y)$. By Lemma 4.4,

$$
u(y)=u\left(x_{\theta}\right) \geq \theta u(x)=\frac{|y-P|}{|x-P|} u(x), \text { hence } \frac{u(y)}{|y-P|} \geq \frac{u(x)}{|x-P|} .
$$

Since $|x-P|=|x|-1$ and $|y-P|=|y|-1$, Lemma 4.1(i) implies equality in these equations. Since $x$ and $y$ are arbitrary, $u(z)=L(\omega)(|z|-1)$ for all $z \in T(\omega)$.

We set $\Omega_{a}=\{x \in \Omega: u(x)<a\}$ for $a>0$ and show, using Lemma 4.5, that $\bar{B}(1, O) \cup \Omega_{a}$ is convex. For $\omega \in S^{n-1}(O)$ and $t>1$, set $Q=Q(t, \omega)=O+t \omega$. Define the hyperplane $H_{t}=H_{t}(\omega)=\{x:\langle x-Q, \omega\rangle=0\}$, and the half-planes $H_{t}^{+}=H_{t}^{+}(\omega)=\{x:\langle x-Q, \omega\rangle>0\}$ and $H_{t}^{-}=H_{t}^{-}(\omega)=\{x:\langle x-Q, \omega\rangle<0\}$. Then $\bar{B}(1, O) \subset H_{t}^{-}(\omega)$. For $a>0$, let $t(a)=t(a, \omega)=1+a / L(\omega)$, and let $Q_{a}=Q_{a}(\omega)=O+t(a) \omega$.

Lemma 4.6. For $a>0$, let $S(a)=\Omega_{a} \cup \bar{B}(1, O)$. Then

(i) $u(x) \geq L(\omega)(\langle x, \omega\rangle-1) \geq L(\omega)(t-1)$ for all $x \in H_{t}^{+}(\omega)$,

(ii) $S(a)=\bigcap_{\omega \in S^{n-1}} H_{t(a)}^{-}(\omega)$ and $H_{t(a)}(\omega)$ is a supporting hyperplane to $S(a)$ at $Q_{a}$, and $O Q_{a} \perp H_{t(a)(\omega)}$, for all $\omega \in S^{n-1}$. Clearly, $S(a)$ is convex.

Proof. By Lemma 4.5, $u(Q)=L(\omega)(|Q|-1)$ for all $\omega \in S^{n-1}$. Also, $Q_{a}$ lies in $H_{t(a)}(\omega)$, and $u\left(Q_{a}\right)=a$. To prove part (i), set $R(\omega)=\{O+s \omega, s>0\}$ and let $r \geq t$. Fix $x \in H_{r}(\omega) \subset H_{t}^{+}(\omega)$, and choose $P \in R(\omega)$, with $|P|$ large, such that 
$|P|-1>|P-x|$. Then $x$ lies in $B(|P|-1, P)$, and by applying monotonicity (2) along the ray $P x$, we see that

$$
L(\omega)=u(P) /(|P|-1) \leq \lim _{|P| \rightarrow \infty} u(x) /(|P|-1-|x-P|)=u(x) /(r-1)
$$

for $P \in R(\omega)$. Since $r=\langle x, \omega\rangle>t$, part (i) follows. We now prove part (ii). Fix $\omega$. Then, by Lemma 4.5, $u(t \omega)<u\left(Q_{a}\right)=a$ whenever $0 \leq t<t(a)=1+a / L(\omega)$. If $A(a)=\bigcup_{\omega \in S^{n-1}}\{t \omega: 1<t<t(a)\}$, then $u(x)<a$ for all $x \in A(a)$. We show that $\Omega_{a}=A(a)$. Clearly, $A(a) \subset \Omega_{a}$, so suppose $x \notin A(a)$, and set $\omega=x /|x|$. Then $x=s \omega$ for some $s \geq t(a, \omega)$, and $x \in H_{t(a)}(\omega) \cup H_{t(a)}^{+}(\omega)$. By part (i), $u(x) \geq a$ and hence $A(a)=\Omega_{a}$. Also $A(a) \cap\left(H_{t(a)}(\omega) \cup H_{t(a)}^{+}(\omega)\right)=\varnothing$ for all $\omega$, implying that $A(a) \subset H_{t(A)}^{-}(\omega)$. As $x \notin A(a)$ implies that $x \notin H_{t(a)}^{-}(\omega)$ for some $\omega$, $A(a)=\bigcap_{\omega}\left(H_{t(a)}^{-} \backslash \bar{B}(1, O)\right)$. By part (i), $\partial A(a) \cap H_{t(a)}(\omega)=Q_{a}(\omega)$. Thus $S(a)$ is convex and $\partial S(a)=\bigcup_{\omega}\left\{Q_{a}(\omega)\right\}=\bigcup_{\omega}\{(1+a / L(\omega)) \omega\}$. Clearly $H_{t(a)}$ is the supporting hyperplane at every $Q_{a} \in \partial S(a)$. By the definition of $H_{t(a)}$ it follows that $O Q_{a}(\omega) \perp H_{t(a)}(\omega)$ for all $\omega \in S^{n-1}$.

We now show that Lemma 4.6 implies that $\Omega_{a}$ is a ball.

Proof of Theorem 1.2. Let $F: \mathbb{R}^{+} \times S^{n-1} \rightarrow \mathbb{R}^{n}$ by $F(a, \omega)=O+(1+a / L(\omega)) \omega$. Then by Lemmas 4.1 and 4.6, for $a>0$ fixed, $F$ is a bijective Lipschitz map, and $F\left(S^{n-1}\right)=\partial \Omega_{a}$. Thus $\partial \Omega_{a}$ is connected and $\vec{F}(\omega) \perp H_{t(a)}(\omega)$. Let $\omega_{1}, \omega_{2} \in S^{n-1}$, then $Q_{1}=Q_{a}\left(\omega_{1}\right)$ and $Q_{2}=Q_{a}\left(\omega_{2}\right)$ lie on $\partial \Omega_{a}$. Let $\Pi$ be the two-dimensional plane containing $O, \omega_{1}$ and $\omega_{2}$, and $C$ be $\partial B(1, O) \cap \Pi$. Note that $Q_{1}$ and $Q_{2}$ lie in $\Pi$. Let $\tau(s) \in \partial B(1, O) \cap \Pi$ be a smooth parametrization of $C$ such that $\tau(0)=\omega_{1}$ and $\tau(1)=\omega_{2}$. The curve $\sigma(s)=F(\tau(s))=(1+a / L(\tau(s))) \tau(s)$ in $\Pi \cap \partial \Omega_{a}$ is Lipschitz continuous in $s$, and $\sigma(0)=Q_{1}$ and $\sigma(1)=Q_{2}$. Let $s_{0} \in[0,1]$ be a point of differentiability of $\sigma(s)$. Call $\Sigma(s)=H_{t(a)}(\tau(s))$; by Lemma $4.6, \Sigma\left(s_{0}\right)$ is the supporting hyperplane at $\sigma\left(s_{0}\right)$. Furthermore, $\Sigma\left(s_{0}\right)$ is perpendicular to $\sigma\left(s_{0}\right)$, and $\sigma(s) \in H_{t(a)}^{-}\left(\tau\left(s_{0}\right)\right) \cap \Pi$ for all $s$. Since $s_{0}$ is a point of differentiability, a simple argument shows that $\sigma^{\prime}\left(s_{0}\right)$ lies in $\Sigma\left(s_{0}\right) \cap \Pi$, and hence $\sigma\left(s_{0}\right) \perp \sigma^{\prime}\left(s_{0}\right)$. Thus $\left|\sigma\left(s_{0}\right)\right|^{\prime}=0$. Since this holds for almost every $s \in[0,1]$, Lipschitz continuity implies that $\left|Q_{2}\right|=|\sigma(1)|=|\sigma(s)|=|\sigma(0)|=\left|Q_{1}\right|$. Thus $\Omega_{a}$ is a ball and $L(\omega)=C$ for all $\omega \in S^{n-1}$. The remainder of the proof follows from Lemma 4.5.

\section{The infinite strip $\left\{0<x_{n}<1\right\}$}

Let $\Omega$ be the infinite strip $\left\{x: 0<x_{n}<1\right\}$, let $H(0)=\left\{x: x_{n}=0\right\}$, and let $H(1)=\left\{x: x_{n}=1\right\}$. We assume that $u$ is $\infty$-harmonic, that $u \geq 0$ in $\Omega$, and that $u$ vanishes continuously on $H(0)$ and $H(1)$. For $r>0$, define $D(r)$ to be 
$\left\{x:\left|x^{\prime}\right|_{n-1}<r, 0<x_{n}<1\right\}$, where $x^{\prime}=\left(x_{1}, x_{2}, \ldots, x_{n-1}\right)$ and

$$
\left|x^{\prime}\right|_{n-1}=\sqrt{\sum_{i=1}^{n-1} x_{i}^{2}} .
$$

Set $M(r)=\sup _{D(r)} u(x)$, with the understanding that $M(0)=\sup _{\left\{x^{\prime}=0\right\} \cap \Omega} u(x)$. We set $L(r)=\left\{x \in D(r):\left|x^{\prime}\right|=r, 0 \leq x_{n} \leq 1\right\}$, the lateral boundary of the cylinder $D(r)$. By the maximum principle, $M(r)$ is attained only on $L(r)$. Let $J(r) \in L(r)$ be such that $M(r)=u(J(r))$. Let $C(r, P)$ denote the truncated cylinder $\left\{x:\left|x^{\prime}-P^{\prime}\right|_{n-1}<r, P_{n}<x_{n}<P_{n}+2 r\right\}$. The function $u_{e}$ is the extension of $u$ to all of $\mathbb{R}^{n}$ defined as follows. Set

$$
u_{e}\left(x^{\prime}, x_{n}\right)= \begin{cases}u\left(x^{\prime}, x_{n}\right) & \text { for } 0 \leq x_{n} \leq 1, \\ -u\left(x^{\prime},-x_{n}\right) & \text { for }-1 \leq x_{n} \leq 0,\end{cases}
$$

and extend periodically with period 2 . Then $u_{e}$ is $\infty$-harmonic in $\mathbb{R}^{n}$; see [Bhattacharya 2002].

Step 1. We first observe that there exists a universal constant $K>0$ such that

$$
\min \left(x_{n}(J(r)), 1-x_{n}(J(r))\right) \geq K \quad \text { for all } r>0 .
$$

Let $T=T(r) \in L(r) \cap H(0)$ and consider the cylinder $C\left(\frac{1}{2}, T\right) \subset \Omega$. Since $x_{n}(T)=0$ and $u>0$ in $C\left(\frac{1}{2}, T\right)$, the boundary Harnack inequality (3) with $s=\frac{1}{16}$, $u_{1}=u, u_{2}=x_{n}$ and $z=T+\frac{1}{8} \vec{e}_{n}=\left(T^{\prime}, \frac{1}{8}\right)$ yields

$$
M_{1} \frac{u(z)}{1 / 8} \leq \frac{u(x)}{x_{n}} \leq M_{2} \frac{u(z)}{1 / 8} \text { for all } x \in C\left(\frac{1}{16}, T\right) .
$$

Let $P=\left(T^{\prime}, \frac{1}{2}\right)$. Since $|z-P| / z_{n}=3$, the Harnack inequality implies that $u(z) e^{-3} \leq u(P) \leq u(z) e^{3}$. Thus (17) with new constants $M_{1}$ and $M_{2}$ yields

$$
M_{1} u(P) \leq \frac{u(x)}{x_{n}} \leq M_{2} u(P) \text { for all } x \in C\left(\frac{1}{16}, T\right) .
$$

Let $E(T)=\left\{x:|x-T|_{n-1}<\frac{1}{16}, 0<x_{n}<\frac{1}{2}\right\}$; if $x \in E(T) \backslash C\left(\frac{1}{16}, T\right)$ then $|x-P| / x_{n} \leq 16$ and

$$
u(P) e^{-16} \leq u(x) \leq u(P) e^{16} .
$$

Then (18), with new $M_{1}$ and $M_{2}$, implies that

$$
M_{1} u(P) \leq \frac{u(x)}{x_{n}} \leq M_{2} u(P) \quad \text { for all } x \in E(T),
$$

since $C\left(\frac{1}{16}, T\right) \subset E(T)$. From this we get

$$
M_{1} u(P) x_{n}(J(r)) \leq M(r) \leq M_{2} u(P) x_{n}(J(r)),
$$


since $J(r) \in L(r) \cap E\left(J^{\prime}(r), 0\right)$. Dividing by $u(P)$ we see that $x_{n}(J(r)) \geq 1 / M_{2}$. We argue similarly for $\operatorname{dist}(J(r), H(1))$, and (16) follows. Note that by the Harnack inequality, $M(r)=u(J(r)) \leq e^{(r+1) / K} u(J(0))=e^{(r+1) / K} M(0)$. Hence $M(r)$ cannot grow faster than the exponential rate.

Step 2. We now show that $M(r)$ is at least of the order $r^{c \log r}$, for large $r$ and for some $c>0$. We work with $u_{e}(x)$; for $r>0$, let $T(r)$ denote the line through $J(r)$ parallel to the $x_{n}$-axis. Clearly,

$$
\sup _{\left\{x:\left|x^{\prime}\right|_{n-1}<r\right\}} u_{e}(x)=M(r) \quad \text { and } \quad \inf _{\left\{x:\left|x^{\prime}\right|_{n-1}<r\right\}} u_{e}(x)=-M(r) .
$$

Let $F(r)=\left(J^{\prime}(r), 2-x_{n}(J(r))\right)$. Then $u(F(r))=-M(r)$, since $u_{e}$ arises from the odd reflection of $u$ about $x_{n}=1$. Note that $|J(r)-F(r)| \leq 2(1-K)=\delta$. Since $M(2 r)-u_{e}(x) \geq 0$ in $\left\{x:\left|x^{\prime}\right|_{n-1}<2 r\right\}$, applying the Harnack inequality to $u_{e}(J(r))$ and $u_{e}(F(r))$, we see that $M(2 r)-M(r) \geq e^{-\delta / r}(M(2 r)+M(r))$, and hence that

$$
M(2 r) \geq \frac{e^{\delta / r}+1}{e^{\delta / r}-1} M(r) \quad \text { for } r>0
$$

We employ iteration noticing that $\left(e^{\delta / r}+1\right) /\left(e^{\delta / r}-1\right) \uparrow \infty$ as $r$ increases. Let $\xi>0$, select $R=R(\xi)>0$ such that $\left(e^{\delta / r}+1\right) /\left(e^{\delta / r}-1\right)>\xi$ for all $r>R$. Then (19) implies that $M\left(2^{m} R\right) \geq \xi^{m} M(R)$ and $M(r) \geq(r / R)^{\log \xi / \log 2} M(R) / \xi$. Also $M\left(2^{m+1} \delta\right) \geq M\left(2^{m} \delta\right)\left(e^{1 / 2^{m}}+1\right) /\left(e^{1 / 2^{m}}-1\right)$. Take $N$ large, so that $e^{1 / 2^{k}}-1 \leq 2 / 2^{k}$ for $k \geq N$. Starting an iteration from $N$, we get

$$
\begin{aligned}
M\left(2^{m+1} \delta\right) & \geq\left(\prod_{k=N}^{m}\left(1+\frac{2}{e^{1 / 2^{k}}-1}\right)\right) M\left(2^{N} \delta\right) \geq\left(\prod_{k=N}^{m}\left(1+2^{k}\right)\right) M\left(2^{N} \delta\right) \\
& =\left(\prod_{k=N}^{m} 2^{k}\right)\left(\prod_{k=N}^{m}\left(1+2^{-k}\right)\right) M\left(2^{N} \delta\right) \geq C(N) 2^{m^{2} / 2} M\left(2^{N} \delta\right) .
\end{aligned}
$$

Since $M(r)$ is increasing, the right side is of the order $r^{c \log r}$, for some universal $c>0$.

\section{References}

[Aronsson et al. 2004] G. Aronsson, M. G. Crandall, and P. Juutinen, "A tour of the theory of absolutely minimizing functions”, Bull. Amer. Math. Soc. (N.S.) 41:4 (2004), 439-505. MR MR2083637 Zbl 02108961

[Barles and Busca 2001] G. Barles and J. Busca, "Existence and comparison results for fully nonlinear degenerate elliptic equations without zeroth-order term", Comm. Partial Differential Equations 26:11-12 (2001), 2323-2337. MR 2002k:35078 Zbl 0997.35023 
[Bhattacharya 2002] T. Bhattacharya, "On the properties of $\infty$-harmonic functions and an application to capacitary convex rings”, Electron. J. Diff. Equations 101 (2002), 22 pp. MR 2003j:35126 Zbl 1037.35028

[Bhattacharya 2004] T. Bhattacharya, "On the behaviour of $\infty$-harmonic functions near isolated points”, Nonlinear Anal. 58:3-4 (2004), 333-349. MR 2073529 Zbl 1053.31003

[Bhattacharya et al. 1989] T. Bhattacharya, E. DiBenedetto, and J. Manfredi, "Limits as $p \rightarrow \infty$ of $\Delta_{p} u_{p}=f$ and related extremal problems", Rend. Sem. Mat. Univ. Politec. Torino special issue (1989), 15-68. MR 93a:35049

[Crandall and Evans 2001] M. G. Crandall and L. C. Evans, "A remark on infinity harmonic functions", pp. 123-129 in Proceedings of the USA-Chile Workshop on Nonlinear Analysis (Viña del Mar and Valparaiso, 2000), Electron. J. Differ. Equ. Conf. 6, Southwest Texas State Univ., San Marcos, TX, 2001. MR 2001j:35076 Zbl 0964.35061

[Crandall et al. 1992] M. G. Crandall, H. Ishii, and P.-L. Lions, "User's guide to viscosity solutions of second order partial differential equations", Bull. Amer. Math. Soc. (N.S.) 27:1 (1992), 1-67. MR 92j:35050 Zbl 0755.35015

[Crandall et al. 2001] M. G. Crandall, L. C. Evans, and R. F. Gariepy, "Optimal Lipschitz extensions and the infinity Laplacian”, Calc. Var. Partial Differential Equations 13:2 (2001), 123-139. MR 2002h:49048 Zbl 0996.49019

[Jensen 1993] R. Jensen, "Uniqueness of Lipschitz extensions: minimizing the sup norm of the gradient”, Arch. Rational Mech. Anal. 123:1 (1993), 51-74. MR 94g:35063 Zbl 0789.35008

[Lindqvist and Manfredi 1995] P. Lindqvist and J. J. Manfredi, "The Harnack inequality for $\infty$ harmonic functions", Electron. J. Diff. Equations 5 (1995), 5 pp. MR 96b:35025 Zbl 0818.35033

Received October 21 2003. Revised May 32004.

TILAK BHATTACHARYA

DEPARTMENT OF MATHEMATICS

PURDUE UNIVERSITY

WEST LAFAYETTE, IN 47907

tbhatta@math.purdue.edu 